\title{
Preface to "Understanding volcanic processes through geophysical and volcanological data investigations: some case studies from Italian sites (EGU2019 GMPV5.11 session, COV10 S01.11 session)"
}

\author{
Paola Cusano $^{1}$, Enza De Lauro ${ }^{2}$, Antonietta Esposito ${ }^{1}$, Mariarosaria Falanga ${ }^{3}$, Danilo Galluzzo ${ }^{1}$, and \\ Simona Petrosino ${ }^{1}$ \\ ${ }^{1}$ Istituto Nazionale di Geofisica e Vulcanologia, Sezione di Napoli - Osservatorio Vesuviano, Via Diocleziano 328, \\ 80124 Naples, Italy \\ ${ }^{2}$ Ministery of Education, Universities and Research, Rome, Italy \\ ${ }^{3}$ Università degli Studi di Salerno, Dipartimento di Ingegneria dell'Informazione ed Elettrica e Matematica applicata/DIEM, \\ Fisciano, Italy
}

Correspondence: Paola Cusano (paola.cusano@ingv.it)

Published: 1 June 2021

\begin{abstract}
Volcanic dynamics is driven by the complex interplay between fluid flow (circulation of magmatic and/or hydrothermal fluids) and rock structure (volcano conduits, dykes), the comprehension of which requires both multiparametric monitoring and modelling of relevant physical and chemical processes of the system. Understanding the factors controlling the dynamics of the processes involved in these interactions is necessary to characterize the overall behaviour of a volcano and the eventual transition mechanisms among stationarity, unrest phases and eruptive styles. The starting point in this context is to have high-quality data of several parameters (seismological, geochemical, geodetic, volcanological), acquired both over years of monitoring activity and focused field experiments. Fundamental contributions come from the use of combined multi-parametric datasets and the adoption of innovative analysis techniques and multi-disciplinary approaches.

This Special Issue is addressed to those researchers, who focus their investigations in the field of volcano dynamics. Its main purpose is to shed light on the processes occurring in active volcanic systems over different time scales, with relevant implications for the hazards and the modern monitoring, thus promoting future discussions on this topic. The Issue contains this introducing preface, which describes the Volume aims, and 14 papers, reflecting the main themes. The papers are devoted to the study of some Italian sites, but the proposed approaches are general and therefore applicable to any other volcanic/hydrothermal areas.
\end{abstract}

\section{Introduction}

This Special Issue is the result of a route started in 2018 with the 10th Cities on Volcanoes international conference, held in Naples (Italy) on 2-7 September, where most of the authors of this Preface were conveners for the session S1.11 "Understanding volcanic processes through geophysical and volcanological data investigations" (http://editoria.rm.ingv. it/miscellanea/2018/miscellanea43/, last access: 3 November 2018). The route continued in the following year, 2019, at European Geosciences Union General Assembly, held in Vienna (Austria) on 13-18 April, where some of the authors of this Preface were convenors of the session GMPV5.11 "Fluid-solid interactions in volcanic processes over different time-scales: an investigation through geophysical and volcanological data" (https://meetingorganizer.copernicus.org/ EGU2019/posters/32735, last access: 22 April 2019). In both events, we invited the conference participants to submit any articles relating to the topics of the Special Volume. Its main scope is the understanding of the factors which control the dynamics of interactions between fluid flow (circulation of magmatic or hydrothermal fluids) and rock structure (volcano conduits, dykes), necessary to characterize the overall behaviour of a volcano, as well as the transitions among stationary, unrest and eruptive phases. A particular attention is devoted to the comprehension of the phenomena that require multi-parametric monitoring and modelling of relevant physical and chemical processes of the system. 
Both the convened sessions were characterized by interesting discussions on different aspects concerning the processes occurring within the volcanic systems; how they manifest over different time scales (from seconds to hours, from days to weeks, etc.); how they can be intercepted, throughout the use of instrumentation spanning all the possible frequency bands associated to the known transients or continuous signals (short-period to broadband seismic velocimeters, gravimeters, tiltimeters, strainmeters, etc.); what are the relevant implications for the hazard; how the interplay between the modern monitoring systems and the research should work.

Starting from those experiences, we focalized the Volume on the following main topics:

1. Characterization of the processes occurring in active volcanic systems over different time scales;

2. Use of techniques based on advanced computational methodologies to achieve new insights in the volcano knowledge;

3. Interplay between the monitoring and field experiments, and the connected research based on high-quality data.

\section{Contributions to the Special Volume}

Beyond this Preface, this Issue contains 14 research papers, reflecting the above evidenced themes. Most of the contributions regards Italians volcanoes, from Mt. Vesuvius, in quiescent state, to Campi Flegrei caldera, that is in unrest, up to Stromboli, that is currently in eruption. However, the described approaches and techniques are general and can be applied to any other volcanic systems.

Falanga et al. (2019) proposed a review on the integrated patterns of seismic and deformation observables, with the aim of recovering common relations. The study areas were two volcanic systems that behave in different ways: almost continuous open conduit activity for Stromboli (Italy), and hydrothermal activity and ground deformation for Campi Flegrei (Italy), currently interested by an unrest phase. Standard and innovative seismological techniques were applied in frequency and time domain (spectral analysis and Independent Component Analysis-ICA, Hyvärinen et al., 2001), and tiltmeter, dilatometer and seismological time series were matched. Earth tides synchronize the activity of the two systems over different time scales. In the case of Stromboli, their variations, in a defined frequency band, marked a departure from the equilibrium state on scale of days, while at Campi Flegrei they modulated the variation of seismicity and ground deformation on annual scale. The authors stressed the necessity to better understand the tidal effects on the tiltmeter/seismic recordings, as well as to investigate the interactions among different phenomena (ground deformation, seismic activity, rainfall, earth tides, etc.).
In the study of Petrosino et al. (2020), the crustal response to external excitations of medium/long-period tidal constituents in terms of ground tilting at Campi Flegrei caldera (Italy) was evaluated by applying a polarization analysis (Montalbetti and Kanasewich, 1970) on borehole tiltmeter time series, from 2015 to 2019. Well defined azimuth directions associated to tidal periodicities (diurnal S1, lunar monthly Mm and lunar fortnightly Mf) resulted to be controlled by the local stress field distribution and the rheology. At some sites the polarization was also affected by the seasonal variations related to rainfall. The results found in this paper have two-fold implications. First, the identification of a ground deformation process permitted to define a background, such as tidal and rainfall effects, on which the endogenous volcanic effects superimpose. Second, the behavior of the exogenous sources provided information on the geometry of structural features and on rock proprieties of the surroundings.

The paper of Cusano et al. (2020) illustrated the properties of the hydrothermal/volcanic system of Ischia Island on long (background seismic noise) and short (earthquakes) time scales. The seismo-volcanic source dynamics of the shallow hydrothermal system, in the northern sector of the Island, was explored throughout the analysis of the seismicity recorded pre and post the $M_{\mathrm{d}} 4.0$ earthquake of $21 \mathrm{Au}-$ gust 2017, both in time and frequency domain (Independent Component Analysis, Hyvärinen et al., 2001; energy pattern; polarization properties, Montalbetti and Kanasewich, 1970). The results suggested that noise and earthquakes were ascribable to a common phenomenon of fluid-rocks interaction. The authors supposed that the basic structure of the hydrothermal system oscillates with normal mode at $1-2 \mathrm{~Hz}$, and an increase of the fluid pressure above a certain threshold may have triggered the $M_{\mathrm{d}} 4.0$ earthquake. Then, pressurization/depressurization cycles created ruptures and reactivation of the faults that generate the subsequent seismicity. The increase in the hydrothermal flux would also have enhanced the activation of the second higher mode at $3-4 \mathrm{~Hz}$, otherwise masked by anthropogenic noise.

Petrosino and Cusano (2020) described a detailed analysis performed on the atypical, low frequency seismicity occurred at Mt. Vesuvius in the time interval 2003-2018. Spectral, polarization (Montalbetti and Kanasewich, 1970), 3D-location (Lomax et al., 2000), cross-correlation and cluster analysis (Greenfield et al., 2019) indicated that such events were ascribable to the dynamics of the deep hydrothermal reservoir (2.5-5 km b.s.1.) which induced variations of the fluid pore pressure in the medium, as well as in the physical-chemical properties of the surrounding rocks. The authors classified the atypical earthquakes as Low Frequency (LF) and Long Period (LP), depending if the source mechanism could be ascribed to slow brittle failure in dry rocks or resonance of fluid filled cracks, respectively. The two categories morph into each other according to the physical state and the mechanical properties of the rocks, as well as the location and 
depth of the source volume. Likely the activation of different source mechanisms could be modulated by the temporal evolution of the dynamical state of the volcano.

The work of Berrino and Ricciardi (2020) presented the results of several absolute gravity measurements carried out at the Campi Flegrei active caldera (Italy) over a dense absolute gravity network. The latter was established in 2015, permitting an advanced approach for volcano monitoring. Between June 2015 and October 2019 five field surveys were carried out, in the course of a slow ground uplift phase. The results of the measurements showed an inverse linear correlation between gravity and height changes, indicating a prevailing mass/density change process at shallow depths. Moreover, a time swing of gravity decrease/increase suggested an alternation of emptying/replenishment processes. The absolute gravity data and the associated gravity variations showed a very good agreement with the outcomes of the pre-existing relative gravity network. The integration of absolute and relative measurements confirmed the existence of processes of fluids' recharge/discharge alternation in the Campi Flegrei shallow hydrothermal system. Therefore, the results excluded, at least over a short time scale, the direct effects of deeper magmatic mass movements. The recorded data and their quality demonstrated the reliability of the absolute gravimetric measurements even if acquired with field instruments, inside a densely urbanized area. They also demonstrated the efficiency of the methodology for volcano monitoring, encouraging the use of absolute gravity networks in dynamically active areas.

The article of Caputo et al. (2020) regards the first spectral analyses performed on thermal time series recorded by thermal infrared cameras (TIRNet) installed in the area of Solfatara volcano and Pisciarelli area (Campi Flegrei, Italy) for surveillance purposes. The dataset consisted of daily maximum temperatures from 25 April 2014 to 31 May 2019, acquired by three TIRs, and atmospheric pressure and air temperature time-series. Data pre-processing was carried out in order to remove the seasonal components, by using the STL algorithm (Seasonal Decomposition of Time Series by Loess, Cleveland et al.,1990). Then, a harmonic analysis (Pawlowicz et al., 2002) was performed in order to identify and remove the long-period tidal constituents (mainly fortnightly and monthly). Finally, Power Spectral Density was calculated to check if characteristic periodicities exist for each site. The reliability and significance of the spectral peaks were proved by statistical and empirical methods (Godin, 1972). The authors found that most of the residual periodicities are related to ambient factors, while two specific cyclicality have a possible local endogenous origin.

The purpose of Nardone et al. (2020) was to study the subsoil structure of the Campi Flegrei area by using both spectral ratios and array techniques applied to seismic noise. The authors applied the Frequency-Wavenumber (Lacoss et al., 1969) and Modified Spatial Autocorrelation (MSPAC, Bettig et al., 2001) techniques to the seismic noise recorded by an underground short period seismic array in order to obtain the dispersion curves. Moreover, single phase velocity values of coherent transient signals were retrieved. The inversion of all these curves, jointly with the $H / V$ spectral ratio from a broadband station, permitted to obtain a shear wave velocity model up to $2000 \mathrm{~m}$ depth. The model had a higher detail for the central part of the caldera and was in a good agreement with the stratigraphic information coming from the shallow boreholes and deep wells drilled for geothermal exploration. In active high risk volcanic areas, such as Campi Flegrei, the definition of an accurate velocity model can have significant implications for better understanding the internal dynamics of the volcano, thus improving the interpretation of monitoring observations.

In Madonia (2020), the measuring systems and the criteria for designing a performing network for thermal monitoring of low temperature fumaroles from Italian active volcanoes (Vulcano, Stromboli, Mt. Etna, Mt. Vesuvius) were presented, illustrating useful insights on the evolution of the activity state of these volcanoes. Time variations of the thermal signal, in terms of both short-lasting transients and medium/long term trends, reflected changes in the activity state of the volcanic system, and/or of seismic activity. Theoretical models of heat transfer processes were discussed, highlighting how difficult was to distinguish between conductive and convective mechanisms or calculate heat fluxes. The retrieved temperature anomalies recorded in the fumarole fields, even if qualitative, revealed as proxies of change in the activity state of the monitored volcanoes. The discussed case histories from the Italian volcanoes highlighted that the continuous monitoring and mapping of the low temperature fumaroles gave useful insights on different phenomena, as upward movements of magma inside the feeding systems, fracturing and dyke intrusions, degassing anomalies driven by seismicity both of volcanic and tectonic origins. The abovementioned considerations, jointly with a low-cost monitoring systems, make this observable a useful and convenient tool in volcanic surveillance.

In Galluzzo et al. (2020), spectral analysis has been applied to almost thousand seismic events recorded at Mt. Vesuvius (Italy) in 2018 with the aim to test a new tool for a fast classification of seismic events. The central frequency and shape factor obtained from the spectral moments of order 0,1 , and 2 , for each event at seven seismic stations were estimated. The analyzed events consisted of volcano-tectonic and low-frequency earthquakes and unclassified events (landslides, rockfalls, thunders, quarry blasts, etc.), most of which were of low energy. The results of the analysis showed that volcano-tectonic earthquakes and low-frequency events were easily distinguishable through the spectral moments values, particularly at seismic stations closer to the epicenter. On the other hand, unclassified events showed spectral parameters values distributed in a broad range which overlap both the volcano-tectonic and the lowfrequency events. Since the computation of spectral parame- 
ters is extremely easy and fast for a detected seismic event, it may become a raw analysis tool of fast application on real or near-real time signals, in order to be effective for the classification of seismic events in observatory practice.

Mancini et al. (2020) analysed and discussed the results of the regional program Rad Campania for the monitoring and the assessment of the radon risk. They developed an innovative methodology, based on a geogenic approach and supported by a comprehensive survey of radon measurement performed in soil gas, natural waters, drinking natural water samples and indoor air. The field measurements were carried out in Campania Region (Italy) with the purpose to investigate the peculiarities of the radon issue at a provincial scale and to redact a map of the radon potential from soil as a tool for authorities to recognize critical areas ("Radon prone areas") to monitor. The authors tried to identify a possible general correlation between geological features of the soil and structural characteristics of the buildings. The results evidenced that to map the radon potential from soil cannot be the only tool to manage the radon issue, but the maintenance of the interface soil-building plays a crucial rule. The categorization of buildings (according to the foundation type, materials, number of floors) showed how it was possible to identify a peculiar behaviour for each construction-type and so tried to build up a performance indicator for different kinds of construction, in terms of response to the potential radon from soil.

Tammaro et al. (2021a) provided a suitable resolution model of the sea tides including low frequency (seiches), tidal bands and non-linear tides by investigating the oscillations of relative sea level. They analysed about 10-year-long tide gauge recordings in the Gulfs of Pozzuoli and Naples (Italy). They proposed a method to filter out the volcanotectonic signal (bradyseism) from the tide gauge records by deconvolving it from two records, one collected in the active volcanic area (Pozzuoli) and the other one collected at a tectonically stable station (Naples). They also retrieved the relative mean sea level change in the Gulf of Naples and compared it with the trend found in five tide gauges spread along the Italian coast.

In the framework of the DINEV project (AMRA, Regione Campania PON, Scarpa et al., 2007) started in the Campi Flegrei - Vesuvius volcanic areas (Italy) in 2004, Di Lieto et al. (2021) carried out subsurface deformation monitoring by using six Sacks-Evertson dilatometers. In 2008, they also employed two arrays of $28-280 \mathrm{~m}$ long water-tube tiltmeters in underground tunnels. A relevant strainmeter and tiltmeter data-set has been collected during the recent Campi Flegrei unrest episode. The high sensitivity strainmeters and tiltmeters provided coherent views of deformation at different time scales, detecting transient events on several time scale, with periods from minutes to months, and monotonic uplift episodes with durations of several weeks to a few years. Their location was compatible with a source inferred from long term deformation signals, located beneath Poz- zuoli. The authors proposed a mechanism for the aseismic strain episodes associated with magma growth in reservoirs with occasional pressure relief associated with the leakage of gas. The authors claimed the importance of expanded arrays of tiltmeters and strainmeters operating continuously, which permit to monitor details of magma-transfer and the underlying cause of subsequent seismic activity.

The work of Bellucci Sessa et al. (2021) showed the ability of GIS (Geographical Information System) to manage a great quantity of data. Two GIS technology applications, based on the seismic catalogue of Campi Flegrei (Italy) from 2005 to 2019 were shown, in addition to the creation of a new GeoDatabase. First, the authors showed how to extract a high quality dataset on the basis of location precision parameters, assessing the reliability of the data through geostatistical analyses. In the second application, they individuated the Campi Flegrei swarms and highlight their temporal trend over the last 15 years. In the latter case, the swarm density and energy release maps were produced. The integration of the morphostructural information in the maps allowed to discuss which structures were involved in the seismic activity. The statistical analysis performed on the swarms permitted to detect the increase of background seismicity against a decrease of the swarms in the last years. Beyond the capability of GIS in integrating and fast analyse a great quantity of multidisciplinary data, this paper demonstrated how this system can fundamentally contribute to any study on the origin and nature of seismicity and claimed that GIS represents an indispensable supporting tool to decision in volcanic unrest.

Tammaro et al. (2021b) simulated the ground deformation of Somma-Vesuvius volcano generated by overpressure sources by means of a finite element (FEM) 3D code, with the main scope of evaluate the influence of topography and structural heterogeneity on deformation. The ingredients used for the simulations were: a Mogi-type sources embedded in an elastic linear isotropic medium, with a varying depth; shape and lateral extension of sources constrained by the outcomes of recent seismic topography studies; structural heterogeneity modelled in terms of dynamic elastic parameters calculated in previous works; a high resolution DTM for the subaerial edifice topography. The simulations' results indicated that real topography and structural heterogeneities were fundamental factors which rule the ground deformation, which is one of the most relevant problems in volcano monitoring. The main result was the evidence of a significant deviation from the axially symmetrical model proposed for the displacement. Finally, the authors stated that a better knowledge of deformation patterns can significantly help in the location of monitoring sensors as well as in the design of an efficient geodetic network. 


\section{Conclusions}

In this Preface we gave a brief description of the Special Volume contents, stressing the complexity of the main topic. The Volume does not have the claim to be complete, but surely it is representative of a variety of disciplines, such as seismology, gravimetry, geodesy, thermography, etc. Great attention was devoted to the high quality experimental data and to innovative monitoring techniques. Moreover, the Volume covered several distinct approaches, such as numerical simulations, unconventional and advanced data analyses, modelling of source and propagation media. Each paper gave new insights in the understanding of the current dynamics of the study area, providing useful contributions to the definition of the system state. These contributions could become crucial for the hazard evaluation and management, especially when dealing with systems whose evolution throughout eruptions or unrest is slow or manifests only by weak precursors.

Acknowledgements. We wish to thank all the authors that have contributed with their articles to the realization of the Special Issue. A special thank goes to the referees that have contributed to improve the quality of the papers with their constructive suggestions. We are grateful the Copernicus Publications staff for its professional support. In particular, we have appreciated the courtesy and availability of Anna Wenzel and Svenja Lange. Finally, we wish to thank Francesca Bianco, Director of INGV-Osservatorio Vesuviano, and the University of Salerno (UNISA) to have supported the realization of the Special Issue.

\section{References}

Bellucci Sessa, E., Castellano, M., and Ricciolino, P.: GIS applications in volcano monitoring: the study of seismic swarms at the Campi Flegrei volcanic complex, Italy, Adv. Geosci., 52, 131144, https://doi.org/10.5194/adgeo-52-131-2021, 2021.

Berrino, G. and Ricciardi, G.: Repeated absolute gravity measurements on a dense network at Campi Flegrei - a reliable tool for volcano monitoring, Adv. Geosci., 52, 41-54, https://doi.org/10.5194/adgeo-52-41-2020, 2020.

Bettig, B., Bard, P. Y., Scherbaum, F., Riepl, J., Cotton, F., Cornou, C., and Hatzfeld, D.: Analysis of dense array noise measurements using the modified spatial auto-correlation method (SPAC). Application to the Grenoble area, Boll. Geof. Teorica Appl., 42, 281-304, 2001

Caputo, T., Cusano, P., Petrosino, S., Sansivero, F., and Vilardo, G.: Spectral analysis of ground thermal image temperatures: what we are learning at Solfatara volcano (Italy), Adv. Geosci., 52, 55-65, https://doi.org/10.5194/adgeo-52-55-2020, 2020.

Cleveland, R. B., Cleveland, W. S., McRae, J. E., and Terpenning, I.: STL: A Seasonal-Trend Decomposition Procedure Based on Loess, J. Off. Stat., 6, 3-73, 1990.

Cusano, P., Petrosino, S., De Lauro, E., De Martino, S., and Falanga, M.: Characterization of the seismic dynamical state through joint analysis of earthquakes and seismic noise: the ex- ample of Ischia Volcanic Island (Italy), Adv. Geosci., 52, 19-28, https://doi.org/10.5194/adgeo-52-19-2020, 2020.

Di Lieto, B., Romano, P., Bilham, R., and Scarpa, R.: Aseismic strain episodes at Campi Flegrei Caldera, Italy, Adv. Geosci., 52, 119-129, https://doi.org/10.5194/adgeo-52-119-2021, 2021.

Falanga, M., De Lauro, E., Petrosino, S., and De Martino, S.: Interaction between seismicity and deformation on different time scales in volcanic areas: Campi Flegrei and Stromboli, Adv. Geosci., 52, 1-8, https://doi.org/10.5194/adgeo-52-1-2019, 2019.

Galluzzo, D., Nardone, L., La Rocca, M., Esposito, A. M., Manzo, R., and Di Maio, R.: Statistical moments of power spectrum: a fast tool for the classification of seismic events recorded on volcanoes, Adv. Geosci., 52, 67-74, https://doi.org/10.5194/adgeo52-67-2020, 2020.

Godin, G.: The Analysis of Tides, University of Toronto Press, Toronto, Canada, 264 pp., ISBN 0802017479 978-0-8020-17475,1972

Greenfield, T., Keir, D., Kendall, J. M., and Ayele, A.: Low-frequency earthquakes beneath Tullu Moye volcano, Ethiopia, reveal fluid pulses from shallow magma chamber, Earth Planet. Sc. Lett., 526, 115782, https://doi.org/10.1016/j.epsl.2019.115782, 2019.

Hyvärinen, A., Karhunen, J., and Oja, E.: Independent Component Analysis, John Wiley \& Sons, New York, USA, 2001.

Lacoss, R. T., Kelly, E. J., and Toksoz, M. N.: Estimation of seismic noise structure using arrays, Geophysics, 34, 21-38, 1969.

Lomax, A., Virieux, J., Volant, P., and Berge, C.: Probabilistic earthquake location in 3D and layered models: Introduction of a Metropolis-Gibbs method and comparison with linear locations, in: Advances in Seismic Event Location, edited by: Thurber, C. H. and Rabinowitz, N., 101-134, Kluwer, Amsterdam, the Netherlands, 2000.

Madonia, P.: Variations of low temperature fumaroles as a tool for detecting changes in volcanic activity state: a brief overview, Adv. Geosci., 52, 97-103, https://doi.org/10.5194/adgeo-52-972020, 2020.

Mancini, S., Guida, M., Cuomo, A., and Guida, D.: A geogenic approach for the Radon monitoring and the exposure assessment at a regional scale: The results of the Rad_Campania project, Adv. Geosci., 52, 87-96, https://doi.org/10.5194/adgeo-52-872020, 2020.

Montalbetti, J. F. and Kanasewich, E. R.: Enhancement of teleseismic body phase with a polarization filter, Geophys. J. R. Astr. Soc., 21, 119-129, https://doi.org/10.1111/j.1365246X.1970.tb01771.x, 1970.

Nardone, L., Esposito, R., Galluzzo, D., Petrosino, S., Cusano, P., La Rocca, M., Di Vito, M. A., and Bianco, F.: Array and spectral ratio techniques applied to seismic noise to investigate the Campi Flegrei (Italy) subsoil structure at different scales, Adv. Geosci., 52, 75-85, https://doi.org/10.5194/adgeo-52-75-2020, 2020.

Pawlowicz, R., Beardsley, B., and Lentz, S.: Classical tidal harmonic analysis with error analysis in MATLAB using T_TIDE, Comput. Geosci., 28, 929-937, 2002.

Petrosino, S. and Cusano, P.: Low frequency seismic source investigation in volcanic environment: the Mt. Vesuvius atypical case, Adv. Geosci., 52, 29-39, https://doi.org/10.5194/adgeo-5229-2020, 2020. 
Petrosino, S., Ricco, C., De Lauro, E., Aquino, I., and Falanga, M.: Time evolution of medium and long-period ground tilting at Campi Flegrei caldera, Adv. Geosci., 52, 9-17, https://doi.org/10.5194/adgeo-52-9-2020, 2020.

Scarpa, R., Amoruso, A., Crescentini, L., Romano, P., De Cesare, W., Martini, M., Scarpato, G., Linde, A. T., and Sacks, I. S.: New borehole strain system detects uplift at Campi Flegrei, EOS Trans. AGU, 88, 197-203, 2007.

Tammaro, U., Obrizzo, F., Riccardi, U., La Rocca, A., Pinto, S., Brandi, G., Vertechi, E., and Capuano, P.: Neapolitan volcanic area Tide Gauge Network (Southern Italy): Ground Displacements and Sea-Level Oscillations, Adv. Geosci., 52, 105-118, https://doi.org/10.5194/adgeo-52-105-2021, 2021a.
Tammaro, U., Riccardi, U., Romano, V., Meo, M., and Capuano, P.: Topography and structural heterogeneities in surface ground deformation: a simulation test for Somma-Vesuvius volcano, Adv. Geosci., 52, 145-152, https://doi.org/10.5194/adgeo-52145-2021, 2021b. 